\title{
Lentiviral-based approach for the validation of cancer therapeutic targets in vivo
}

\author{
Chiara Ambrogio ${ }^{1}$, Patrick Stern², Claudio Scuoppo ${ }^{3}$, Harald Kranz ${ }^{4}$, Mariano Barbacid ${ }^{1}$, \\ and David Santamaría ${ }^{1}$ \\ ${ }^{1}$ Molecular Oncology Programme, Centro Nacional de Investigaciones Oncológicas (CNIO), Spanish \\ National Cancer Research Center, Madrid, Spain, ${ }^{2}$ Sextant Therapeutics Inc, Cambridge, MA and \\ Koch Institute for Integrative Cancer Research, MIT, Cambridge, MA, ${ }^{3}$ Institute for Cancer Genetics \\ and Herbert Irving Comprehensive Cancer Center, Columbia University, New York, New York, and \\ ${ }^{4}$ Gene Bridges, GmbH Commercial Centre, Heidelberg, Germany
}

BioTechniques 57:179-187 (October 2014) doi 10.2144/000114214

Keywords: lentiviral delivery; target validation; cancer mouse model

Supplementary material for this article is available at www.BioTechniques.com/article/114214/

Despite the pressing need for novel cancer treatments, our improved understanding of tumor biology is not being successfully translated into better therapies. Here we present a lentiviral vector that enables in vivo validation of cancer therapeutic targets when combined with existing cancer animal models that faithfully reproduce the natural history of human disease. Unlike the conventional genetic approaches with targeted alleles, the outlined experimental strategy could be used to assess the preclinical efficacy of a growing number of putative therapeutic hits in a rapid and cost-effective manner.

In recent years, the advent of genomic manipulation of mice has facilitated the production of genetically engineered mouse models (GEMMs) that realistically recapitulate human cancer (1). In particular, the use of site-specific recombinases has facilitated the spatiotemporal mutation of the genome, allowing for controlled inactivation of tumor suppressors and/or induction of endogenous oncogenes (2). Thus, activation of the oncogenic alteration occurs in adult mice, is restricted to limited numbers of selected cell populations, and is subject to the control of endogenous regulatory elements. By virtue of these features, the originating tumors faithfully mimic the progression of human disease. These models offer an in vivo scenario amenable to experimental manipulation, providing an invaluable tool for the identification of the molecular mechanisms that initiate and sustain cancer growth.

GEMMs have also played a key role in advancing customized cancer treatment by facilitating the validation of potential therapeutic targets by genetic means. However, although conventional genetic validation of putative targets based on the ablation of endogenous alleles is a robust approach, it is also a tedious procedure that is far from being a cost- or timeeffective alternative. Hence, there is an urgent demand for the development of a versatile, regulatable, and economical gene targeting procedure to allow tumor-specific target validation in vivo. To this end, we have combined in a single lentiviral vector all of the functional elements required to trigger tumor formation and perform preclinical target validation in any murine conditional cancer model reliant on the Cre-recombinase activity with the additional requirement of a resident reverse Tet-transactivator (rtTA) transgene. Furthermore, we have incorporated insulator genetic elements to prevent epigenetic silencing of the viral sequences, a phenomenon of great concern when performing longlasting in vivo experiments.

\section{Materials and Methods \\ Ethics statement}

All animal experiments were approved by the CNIO Ethical Committee (Permit Ref \# PA-431-08) and performed in accordance with the guidelines stated in The International Guiding Principles for Biomedical Research Involving Animals, developed by the Council for International Organizations of Medical Sciences (CIOMS).

\section{METHOD SUMMARY}

We have devised a lentiviral system to be used in combination with any of the widely used conditional oncogenic mouse models reliant on the Cre/lox technology. Our approach allows controlled and independent induction of both the initiating oncogene as well as regulatable and homogeneous shRNA-mediated knockdown of the potential therapeutic target. This is facilitated by the incorporation of functional DNA insulators that prevent epigenetic silencing, an essential feature for long-term in vivo experiments. 
Plasmid assembly by Red/ET recombination

Plasmid pLB2 (3) was chosen as the backbone for assembly of pTOSCA and modified stepwise by Red/ET recombination $(4,5)$. The technology relies on in vivo homologous recombination mediated by the Rac-phage recE and recT genes or the phage lambda red $\alpha$ and red $\beta$ genes (6) and requires, in contrast to other technologies, no specific recombination sites. It is therefore possible to insert functional elements at virtually every position in a large plasmid. Typically, Red/ET recombination occurs between a linear and a circular reaction partner using homology arms of approximately $50 \mathrm{bp}$ in length (7), which are conveniently attached to the linear reaction partner by polymerase chain reaction (PCR).

For the majority of the steps, Red/ ET recombination was performed according to a standard protocol of
Gene Bridges GmbH (Heidelberg, Germany). Briefly, PCR fragments were amplified from different plasmids carrying the corresponding functional element of choice and combined with a selectable marker gene by another PCR reaction. The obtained amplicons were gel purified. Bacterial cells harboring $\mathrm{pRed} / \mathrm{ET}$ and the circular plasmid in which the functional element should be integrated were cultured aerobically in $1.4 \mathrm{~mL}$ of Luria-Broth (LB) medium at a temperature of $30^{\circ} \mathrm{C}$. At an $\mathrm{A}_{600}$ of 0.3 , expression of red genes was induced by the addition of $50 \mu \mathrm{l} 10 \%(\mathrm{w} / \mathrm{v})$ arabinose and the temperature was increased to $37^{\circ} \mathrm{C}$ to ensure maximal expression and activity of the recombination proteins and prohibit replication of $\mathrm{pRed} / \mathrm{ET}$. After 45 min, cells were sedimented, washed twice with ice-cold 10\% (v/v) glycerol, and electroporated with 100 ng of the corresponding PCR product. Samples from which L-arabinose was omitted were used as negative controls.

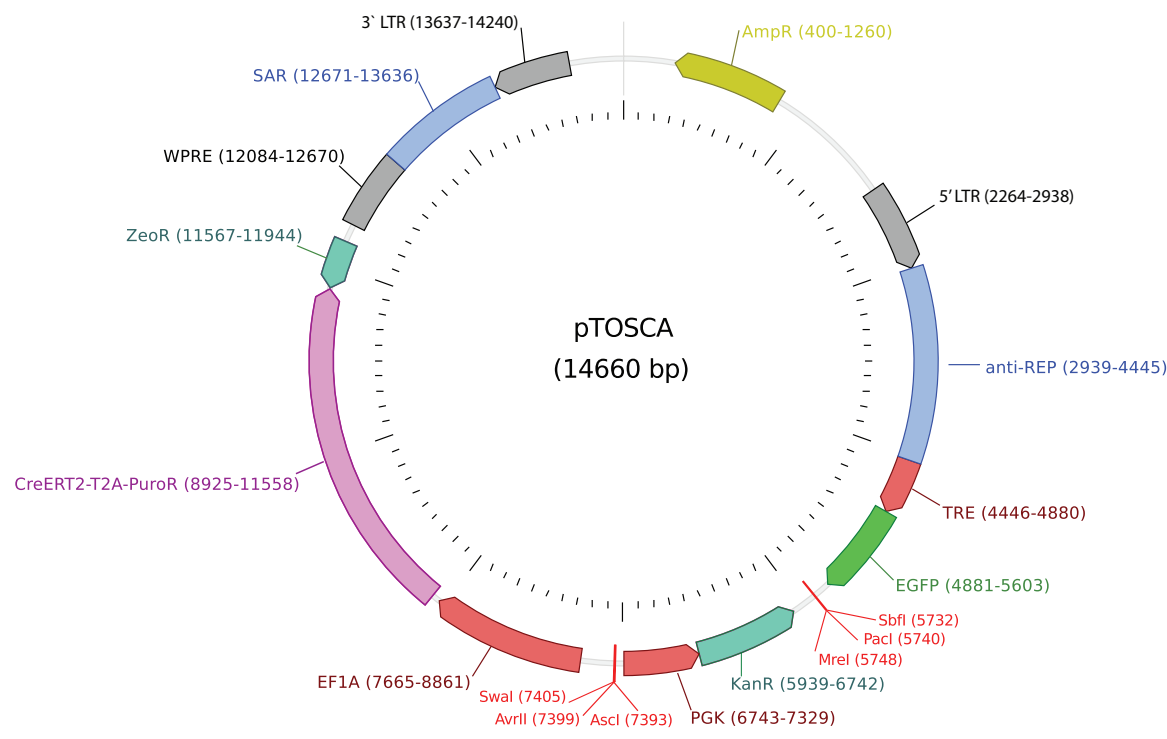

Figure 1. Schematic representation of the lentiviral plasmid pTOSCA (tetracycline operated silencing and Cre-ERT2 activation) constituents. A doxycycline-responsive promoter (TRE, tet-responsive element) controls the expression of a fluorescent spacer (EGFP) together with the shRNAmir30 that is inserted by replacing the kanamycin resistance marker $(K a n R)$ with the indicated restriction enzymes. The zeocin resistance gene $(Z e o R)$ is included as a second selectable marker to diminish backbone recombination and ensure plasmid stabilization after the replacement of KanR by the shRNA. The constitutive EFIA promoter drives expression of the tamoxifen-inducible Cre recombinase (Cre-ERT2) together with the puromycin resistance marker (PuroR) as a bicistronic unit by means of the T2A peptide. Upon long terminal repeat (LTR)-mediated integration into the host genome, the plasmid backbone is flanked by the genetic elements scaffold attachment region (SAR) and antirepressor (anti-REP) to minimize epigenetic silencing. The region in between LTR sequences contains elements for plasmid amplification in bacteria (replication origin and ampicillin resistance). WPRE: woodchuck hepatitis virus posttranscriptional regulatory element.

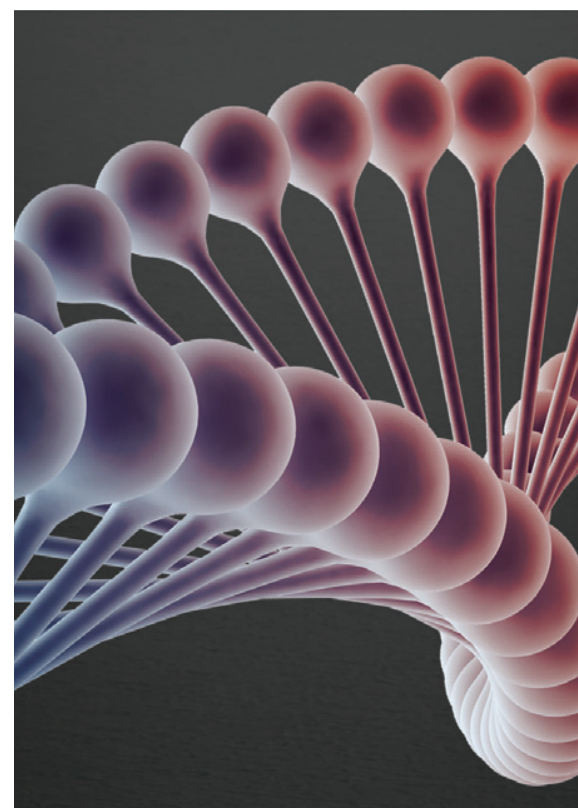

\section{choose a new} way of doing

\section{agarose}

- Convenience

- No boilovers

- No mess
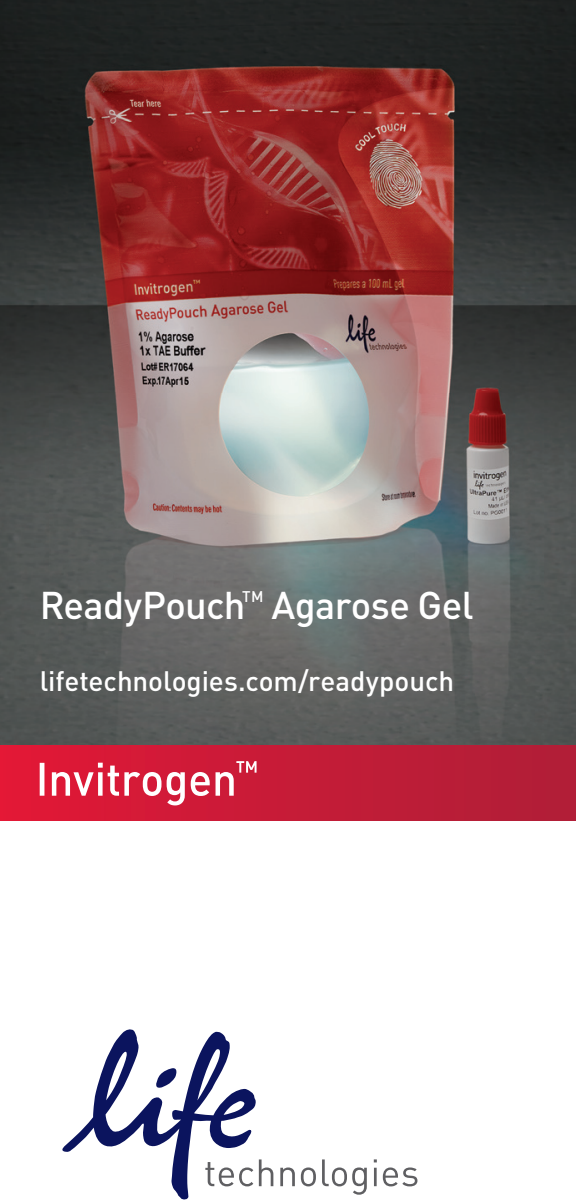

A Thermo Fisher Scientific Brand 
After electroporation, cultures were plated on LB agar containing appropriate antibiotics for the selection of the modified plasmid. A combination of Red/ET recombination and unique restriction site elimination was used when necessary (8). Functional elements were obtained from the following plasmids: TRE-EGFP-miRNA cassette from plasmid pTGM (Plasmid \#32716, Addgene, Cambridge, MA); CreERT2 from Gene Bridges (product \#A013); FRT-PGK-gb2-neo-FRT from Gene Bridges (product \#A002); and T2A-puro-zeo, a combined building block from plasmid pCAGGS-FLPe (Gene Bridges product \#A201) and a synthesized fragment.

\section{shRNA sequence prediction} and subcloning

To identify optimal small hairpin RNA (shRNA) triggers, first we defined the target sequence as the set of mRNA sequences common to all target isoforms as determined by the Refseq IDs of the intended target. All possible 21-nucleotide small interfering RNA (siRNA) sequences were then generated and scored according to the method of Vert and colleagues (9), and the top $5 \%$ were used for the steps that follow. Next, we defined a linear predictor score (LPS) for each sequence by combining features, including (i) relative content of each nucleotide, (ii) any nucleotide repeat or combination of repeats, and (iii) 5'-to-3' sequence asymmetry. The LPS distribution of efficient shRNAs was based on experimentally validated shRNAs and shRNAs scoring in mir30based RNAi screens (10-12). Control sequences included all nonscoring shRNAs targeting the same genes for which the experimentally validated shRNAs described were available. Only features enriched at least 2-fold in the efficient shRNAs set compared with the nonscoring shRNAs set were included in the LPS. A probability for efficient shRNA was then calculated based on the LPS distribution of efficient shRNAs according to Bayes' rules. The resulting shRNAs $(P>0.8)$ were embedded in the mir30 backbone as previously described (13) with the corresponding modifications to incorporate the recognition sequences for Sbf1 and Asc1 restriction enzymes at the $5^{\prime}$ and $3^{\prime}$
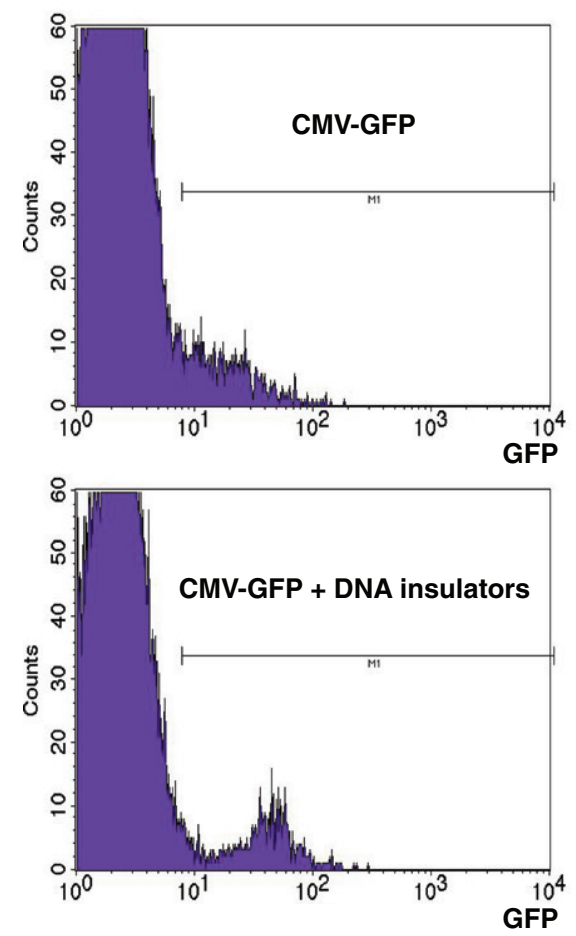

\begin{tabular}{|c|c|}
\hline Lentiviral backbone & MFI \pm SD \\
\hline CMV-GFP & $18 \pm 10$ \\
\hline $\begin{array}{c}\text { CMV-GFP + DNA } \\
\text { insulators }\end{array}$ & $33 \pm 8$ \\
\hline
\end{tabular}

Figure 2. Evaluation of DNA-insulating properties in vivo. $\mathrm{CD} 4^{+} \mathrm{T}$ cells expressing GFP were generated by lentiviral infection of bone marrow and reconstitution into irradiated hosts. T cells were analyzed by FACS eight weeks after reconstitution for GFP expression in the absence and presence of DNA insulating elements as indicated. Mean fluorescence intensity (MFI) is shown \pm standard deviation. CMV: cytomegalovirus.

ends, respectively. The sequence of the shRNA targeting $C$-Raf was as follows: 5'- TGCTGTTGACAGTGAGCGATGGCATGGAGATGTTGCAGTATAGTGAAGCCACAGATGTATACTGCAACATCTCCATGCCACTGCCTACTGCCTCGGA-3'.

In vitro validation

Lentiviral particles were produced in 293T packaging cells, concentrated by ultracentrifugation, and titrated as previously described (14). Mouse embryonic fibroblasts (MEFs) constitutively expressing the rtTA transactivator were infected, selected with 2 $\mu \mathrm{g} / \mathrm{mL}$ puromycin for $72 \mathrm{~h}$, and grown with or without $1 \mu \mathrm{g} / \mathrm{ml}$ doxycycline for an additional $72 \mathrm{~h}$. Cells were harvested and processed for Western blotting procedures as previously described (15) or analyzed by FACS to monitor GFP induction as a surrogate marker of micro RNA (miRNA) expression.

In vivo validation

K-Ras ${ }^{\text {LSLG12Vgeo }}$ and Rosa26 $6^{\text {LSLrtTA }}$ mice have been previously described $(16,17)$. Mice were infected intra-tracheally with $10^{4}$ viral particles as previously described (15). Cre-mediated induction of the K-Ras ${ }^{\mathrm{G} 12 \mathrm{~V}}$ oncogene was induced by intraperitoneal administration of 1 mg of 4-hydroxytamoxifen (4OHT) as described (16). When indicated, mice were fed ad libitum a doxycycline containing diet (D12100401, Research Diets, New Brunswick, NJ) for the entire duration of the experiment. Histopathologic analysis, immunostaining, and quantification of tumor burden were performed on lung serial sections as described (15). All strains were genotyped by Transnetyx (Cordova, TN).

\section{Results and discussion}

Here we set out to optimize a rapid and flexible method for the in vivo validation of putative cancer therapeutic targets. To this end, we devised a lentiviral-based approach that allows controlled and independent induction of both the initiating cancer event and the knockdown of potential therapeutic targets. We have selected the site-specific recombinase Cre/lox system as a tumor-promoting tool because it has been widely used to trigger cancer initiation in GEMMs by ablation of tumor suppressors; activation of endogenous oncogenes; or induction of chromosomal translocations, deletions, or inversions (2). In our system, tumor initiation and target silencing are both achieved by means of a lentiviral construct developed as a recipient vector for any miR30-based shRNA together with an expression cassette containing the Cre recombinase (Figure 1). We decided to use a tamoxifen-inducible Cre version (Cre-ERT2) because it has been shown to lack toxic side effects at the cellular level compared with constitutive expression of the conventional Cre recombinase (18). As the second constituent of the lentiviral module, we used a doxycycline-inducible miR30based shRNA expression cassette 


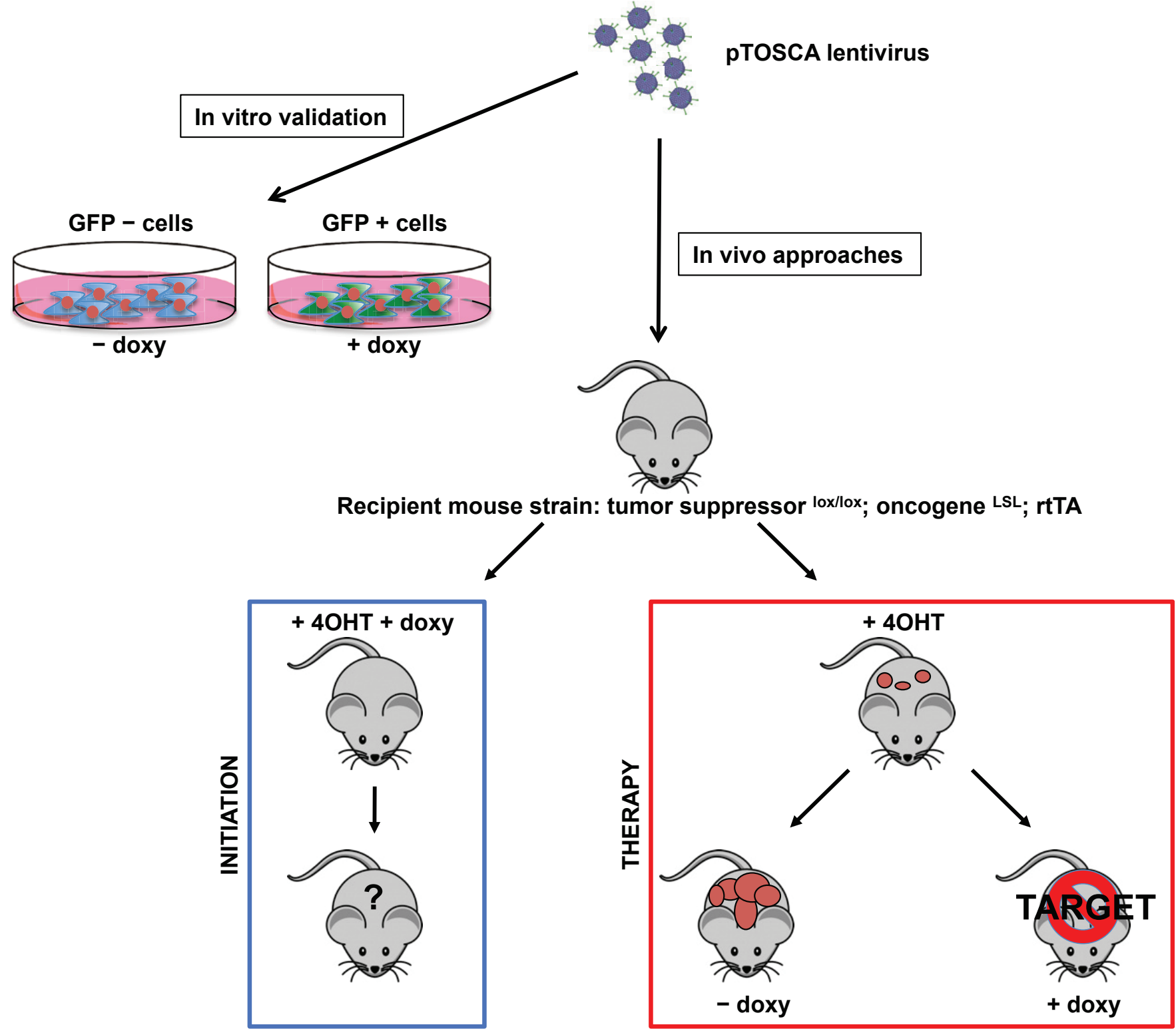

Figure 3. Outline of the target validation procedure. Upon subcloning of the shRNA candidate sequences, the plasmid is used to generate lentiviral particles following standard methodology. Infection of rtTA-expressing cells is used for the evaluation of the most effective sequences; expression of a fluorescent spacer (EGFP) is used as a surrogate marker to confirm infection efficiency. The selected lentiviral particles are used to infect mice carrying an rtTA knock-in together with a Cre/lox-inducible cancer-initiating genetic element. This could be a silent knock-in oncogenic mutation controlled by a floxed transcriptional stop cassette (LSL) or a tumor suppressor flanked by loxP sites. The choice of infection route may vary depending on the tumor type. The doxycycline (doxy) regimen can be adjusted to assess the role of the putative target during tumor initiation (left) as well as to evaluate its therapeutic efficacy (right). 4OHT: 4-hydroxytamoxifen.

linked to a fluorescent reporter that has been effectively harnessed to induce target knockdown in vivo (19). Finally, epigenetic silencing of viral constructs is a serious concern when the in vivo approach relies on consistent and long-term expression of a particular transcript. Silencing is of particular concern in conditions in which specific genetic manipulations may confer a selective disadvantage. To counteract this problem, we flanked our expression cassette with two DNA-insulating elements that have been shown to confer positionindependent and long-term transgene expression in vivo $(3,20)$. We decided to incorporate two DNA insulators that, when inserted into a lentiviral vector, increased the penetrance and expression of a GFP transgene from 20\% (no insulator control) to approximately $70 \%$ after 5 generations (3). To further assess the advantage conferred by these DNA-insulating elements, we purified Lin- bone marrow stem cells and infected them with GFP-expressing lentiviral particles followed by injection into irradiated syngeneic recipient mice. Eight weeks after reconstitution, spleen cells were analyzed for GFP expression by FACS. Remarkably, the presence of both DNA insulators resulted in a significant increase of the mean fluorescence intensity of splenic $\mathrm{CD} 4^{+} \mathrm{T}$ cells (Figure 2). It has been recently reported that long-term transgene expression can be achieved upon lentiviral delivery in vivo $(21,22)$. However, because of 
A

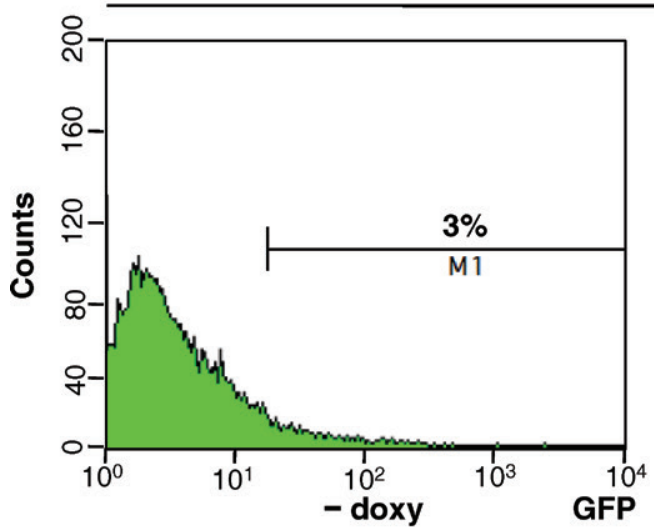

MEFs rtTA

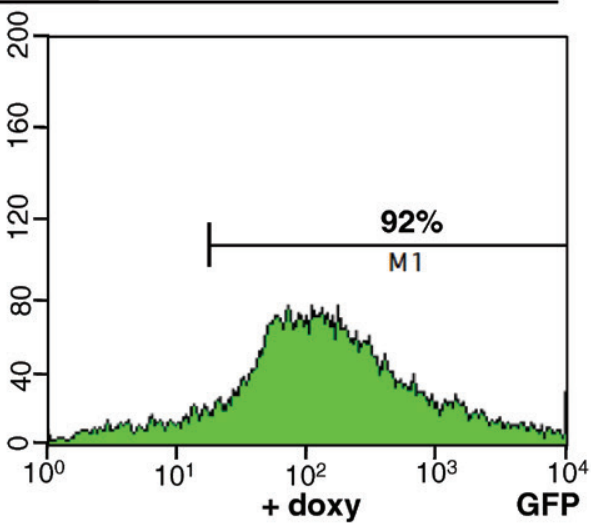

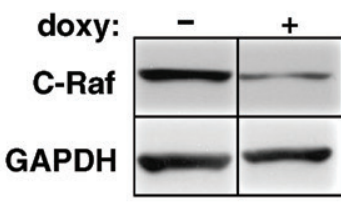

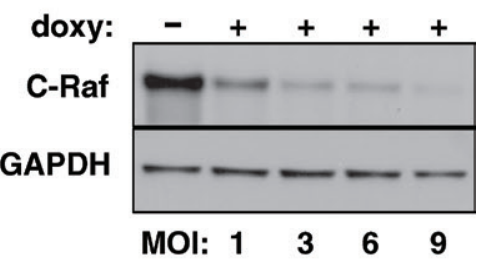

B

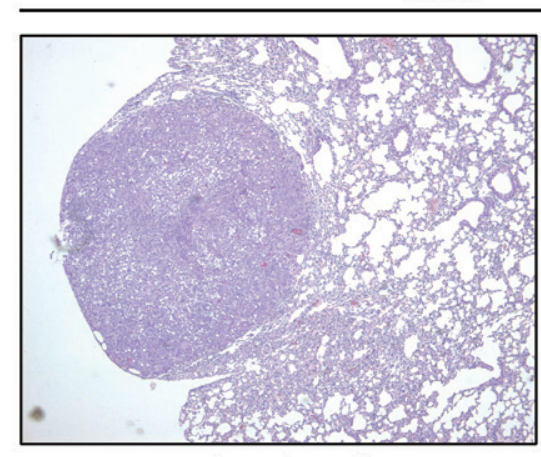

$-\operatorname{doxy}(n=4)$
KRas'LLG12v; Rosa26 LSLITA

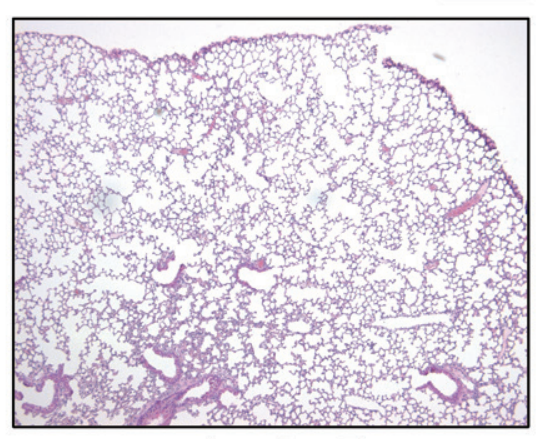

$+\operatorname{doxy}(n=7)$
C

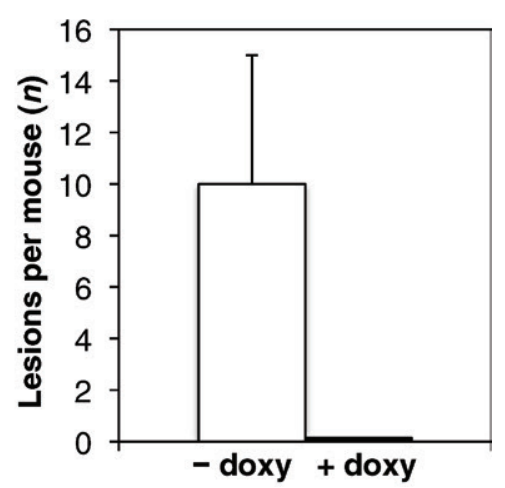

Figure 4. Lentiviral-mediated validation of C-Raf kinase as an essential mediator in $\boldsymbol{K}$-Ras $\boldsymbol{s}^{\mathbf{G} 12 \mathrm{v}}$-driven non-small cell lung carcinoma. (A) Infection of $r$ tTA-ex-

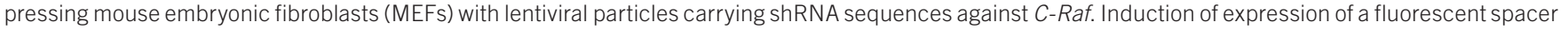
(EGFP) as well as C-Raf knockdown efficacy were assessed by FACS and Western blotting after three days in the presence of doxycycline (doxy; upper West-

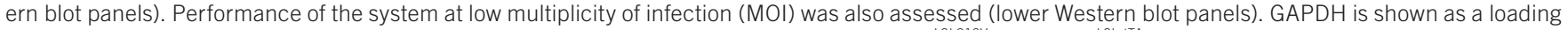

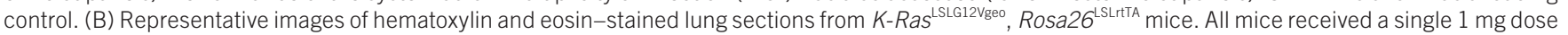

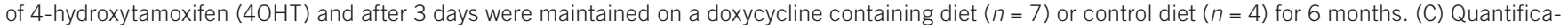
tion of the number of lesions six months after the lentiviral infection in controls and doxycycline-treated cohort. Data are shown as mean \pm standard deviation.

the random integration of the lentiviral backbone, local transcription control can have a mixed effect on expression of the integrated construct, resulting in a high degree of variation. The insulator elements help to maintain long-term expression and diminish local variability (Figure 2). For these reasons, the presence of the insulator sequences may be advantageous in applications subject to negative selection as in the case of validation of putative cancer therapeutic targets.

We next decided to test the efficacy of the lentiviral approach in an aggressive tumor model (the routine experimental layout is shown in Figure 3). To this end, we focused on the K-Ras oncogene that is present in over 30\% of all human non-small cell lung cancer (NSCLC). Furthermore, unlike other subtypes of NSCLC driven by alternative oncogenes such as mutant epidermal growth factor receptor or EML4-ALK, currently there is no targeted therapy to treat $K$-Ras mutant patients. Thus, we selected a K-Ras-driven cancer model as a proof of concept to evaluate our target validation set-up in vivo. We have used an inducible $K$ - Ras $^{\text {G12V }}$ strain engineered as a knock-in mutation within the endogenous locus that is maintained silent by a preceding floxed transcription stop cassette (Lox-StopLox or LSL). Activation of the endogenous K-Ras mutant allele is induced upon Cre-mediated excision of the LSL cassette, resulting in the development of NSCLC (16).

Following a conventional genetic approach, C-Raf has been recently shown to be an essential mediator of oncogenic K-Ras in NSCLC $(15,23)$. Therefore, we decided to use the C-Raf kinase as a contingent therapeutic target to evaluate the lentiviralmediated methodology. Optimal shRNA sequences targeting the $\mathrm{C}$-Raf kinase were generated by a classifier based on balanced voting between four machinelearning tools, which were trained on experimentally validated shRNAs (24) and shRNAs scoring in several mir30based RNAi screen (C.S., manuscript in preparation). The lentiviral construct carries puromycin $N$-acetyl-transferase as a selectable marker downstream of the Cre-ERT2 and linked by the $2 \mathrm{~A}$ peptide that facilitates the synthesis of multiple gene products from single transcripts. This allowed in vitro selection of the most effective shRNA sequences upon infection of cells 
expressing rtTA (Figure 4A). Importantly, efficient knockdown of the target was achieved at a multiplicity of infection (MOI) of 1 , demonstrating single copy efficacy, thus reinforcing the notion that this is a feasible approach in vivo (Figure 4A). K-Ras ${ }^{\text {LSLG12Vgeo }}$, Rosa26 ${ }^{\text {LSLrTA }}$ mice carrying the endogenous $K$-Ras ${ }^{G 12 \mathrm{~V}}$ oncogene as well as an rtTA targeted to the Rosa26 locus (17) were infected intra-tracheally with the selected lentiviral particles. We have used $10^{4}$ viral particles/mouse because this has been previously shown to efficiently induce tumor formation in a similar Cre-dependent model (25). After three days, all animals were subjected $4 \mathrm{OHT}$ injections to activate the Cre-ERT2 recombinase and induce expression of both the endogenous K-Ras oncogene as well as the rtTA. At this point, a cohort of mice was fed a doxycycline-containing diet to induce C-Raf knockdown upon expression of the specific shRNA. The diet was maintained for a period of 6 months to assess the stability and long-term in vivo performance of the system. As expected, histologic charac- terization of the lungs from the control (doxycycline free, $n=4$ ) cohort identified tumor lesions classified as adenocarcinomas in all examined animals. Remarkably, all inspected mice from the doxycycline diet cohort $(n=7)$ were found to be tumor free (Figure 4B). An unrelated shRNA did not interfere with tumor development (data not shown). These tumors were used to assess the homogeneous expression of the shRNA by means of the GFP reporter (Supplementary Figure S1). In sum, these results demonstrate that our shRNAmediated lentiviral strategy is a feasible approach for long-term target validation purposes in vivo.

Over the past two decades, our improved understanding of the biology of cancer has resulted in the identification of molecular and cellular traits that are exclusive to tumor cells. As a consequence, drug development has shifted toward agents that target these specific molecular alterations. The resulting targeted cancer therapies offer tumor-specific selectivity and as a consequence may be more effective than conventional cytotoxic regimens and substantially less harmful to normal cells. Such an approach is particularly relevant for tumor types that remain elusive targets for conventional therapy. Among those, cancers carrying the $K$-Ras oncogene are a paradigmatic example. K-Ras-activating mutations result in aggressive tumors, generally correlate with a poor prognosis, and are associated with mediocre response to many existing treatments (26). Recent therapeutic efforts have used unbiased functional genomics and synthetic lethal screens in search of $K$-Ras-specific pharmacologically tractable targets (27). Actually, comprehensive methods integrating various high-throughput approaches have been implemented $(28,29)$, and the concerted outcome of all these efforts has provided a significant number of putative therapeutic targets that may eventually join the pipeline of candidates in need of pharmacologic development. Ideally, these targets should formerly undergo rigorous functional validation in vivo. This is an important issue because

\section{UNDER WRAPS:
Cell Culture Overhaul}

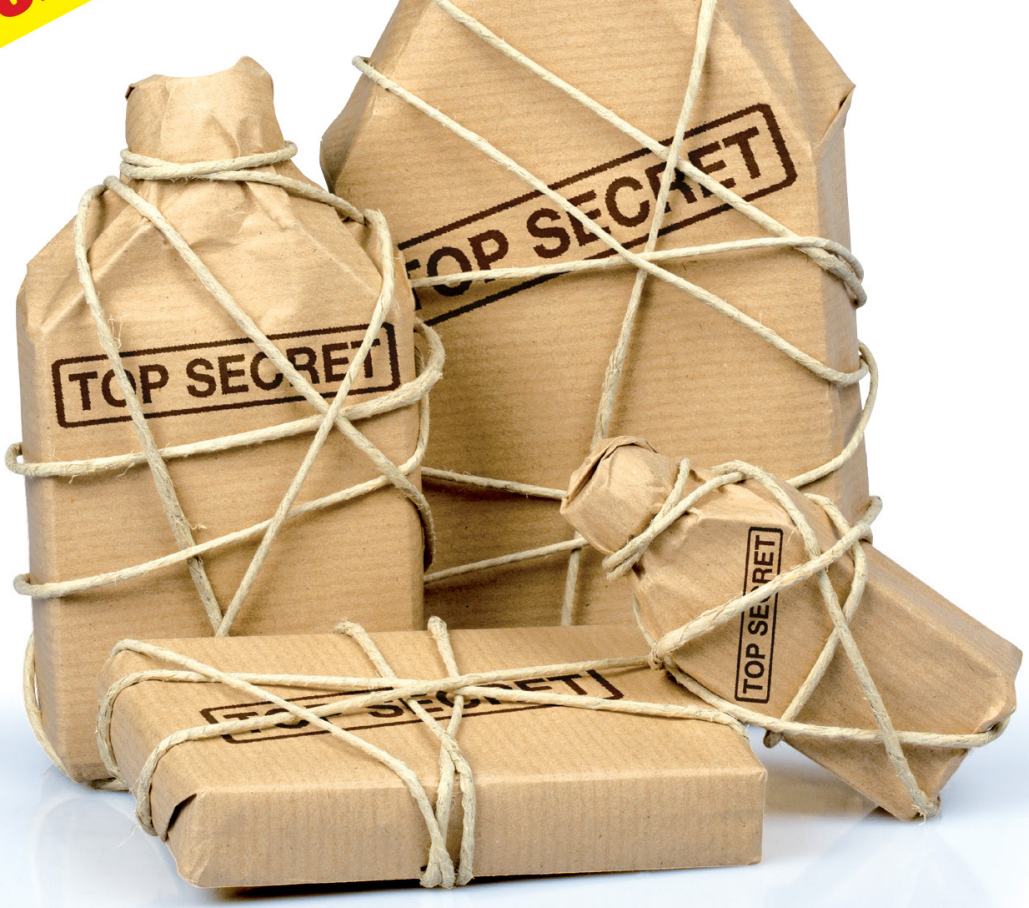

After 20 years, it was time for an update.

We've painstakingly

- redesigned

- expanded

- optimized 
many candidate therapeutic targets have ultimately failed to induce stable tumor remission when translated to clinical trials (30). We believe that the lentiviral approach described here will facilitate a rapid and cost-effective in vivo validation of putative therapeutic targets in a number of aggressive tumor types. Other delivery routes could be used for the induction of tumors in tissues other than lung (21).

In addition to the target validation method outlined earlier, several other applications could be implemented based on minor modifications of the existing viral backbone subject to lentiviral packaging limits. For instance, expression of additional cDNAs could be achieved by converting the bicistronic transcript into a multicistronic unit by means of further self-processing 2A peptides. This could be used for the expression and functional evaluation of presumed co-driver mutations. Furthermore, our system is readily compatible with a number of new mir-RNA based improved structures (31). Finally, the emergence of resistance is one of the major hurdles faced by those targeted therapies currently undergoing clinical trials (30). Several applications could be used to investigate and validate mechanisms of drug resistance, both intrinsic and acquired. The lentiviral approach could be combined with already existing pharmacologic treatments to validate candidate mediators of intrinsic resistance. In this setting, the inducible shRNA knockdown could be used to reassess therapeutic efficacy upon inactivation of putative drug efflux transporters, drug modifiers, or antiapoptotic/pro-survival factors. Importantly, the mir30 system enables two shRNAs to be linked together in cis, allowing co-knockdown of two targets to assess combination therapies $(20,32)$. In addition, tumors become treatment refractory by acquired resistance because of secondary mutations. Anticipating the nature of these resistance drivers could allow initial co-targeting of the relevant pathways to enhance therapeutic efficacy. To this end, the lentiviral system can be used to anticipate mechanisms of drug resistance in vivo by performing sequential on-off doxycycline intervals resulting in intermittent target inhibition. This is an advantage compared with permanent gene editing procedures such as CRISP/ Cas technology (33). This alternating regimen mimics clinical practice and has been previously shown to facilitate the selection of mutant drug-resistant tumor subclones (34). This protocol may help the expansion of clones with mutations affecting other proteins that deregulate the driver oncogene or alternative signaling pathways (or both). Both can be subsequently identified by deep sequencing approaches.

\section{Authors contributions}

C.A. and D.S. conceived the study and performed the experiments. P.S. performed reconstitution experiments, provided essential plasmid components and contributed expertise. C.S. designed shRNA sequences and provided valuable expertise. H.K. constructed the final version of the lentiviral vector. C.A. and D.S. wrote the paper with contributions from all co-authors. M.B. supervised the project.

\section{Acknowledgments}

C.A. is the recipient of a postdoctoral fellowship from the Spanish Association Against Cancer (AECC). This work was supported by grants from the European Research Council (ERC-AG/250297-RAS AHEAD), the EU-Framework Programme (HEALTHF2-2010-259770/LUNGTARGET, HEALTH-2010-260791/EUROCANPLATFORM), the Spanish Ministry of Economy and Competitiveness (SAF2011-30173), and the Autonomous Community of Madrid (S2011/ BDM-2470/ONCOCYCLE) to M.B. We are grateful to Scott Lowe (Memorial Sloan Kettering Cancer Center, New York, NY) for providing the inducible miR30-based shRNA expression cassette and for critical reading of the manuscript, and to Saskia Loesch for technical assistance.

\section{Competing interests}

Harald Kranz is an employee of Gene Bridges Gmbh.

\section{References}

1. Kwon, M.C. and A. Berns. 2013. Mouse models for lung cancer. Mol. Oncol. 7:165-177.

2. Frese, K.K. and D.A. Tuveson. 2007. Maximizing mouse cancer models. Nat. Rev. Cancer 7:645-658.

3. Kissler, S., P. Stern, K. Takahashi, K. Hunter, L.B. Peterson, and L.S. Wicker. 2006. In vivo RNA interference demonstrates a role for Nramp1 in modifying susceptibility to type 1 diabetes. Nat. Genet. 38:479-483.

4. Sawitzke, J.A., L.C. Thomason, N. Costantino, M. Bubunenko, S. Datta, and D.L. Court. 2007. Recombineering: in vivo genetic engineering in e. coli, s. enterica, and beyond. Methods Enzymol. 421:171-199.

5. Zhang, Y., F. Buchholz, J.P. Muyrers, and A.F. Stewart. 1998. A new logic for DNA engineering using recombination in Escherichia coli. Nat. Genet. 20:123-128.

6. Muyrers, J.P., Y. Zhang, F. Buchholz, and A.F. Stewart. 2000. RecE/RecT and Redalpha/ Redbeta initiate double-stranded break repair by specifically interacting with their respective partners. Genes Dev. 14:1971-1982.

7. Noll, S., G. Hampp, H. Bausbacher, N. Pellegata, and H. Kranz. 2009. Site-directed mutagenesis of multi-copy-number plasmids: $\mathrm{Red} / \mathrm{ET}$ recombination and unique restriction site elimination. Biotechniques 46:527-533.

8. Vert, J.-P., N. Foveau, C. Lajaunie, and Y. Vandenbrouck. 2006. An accurate and interpretable model for siRNA efficacy prediction. BMC Bioinformatics 7:520.

9. Luo, J., M.J. Emanuele, D. Li, C.J. Creighton, M.R. Schlabach, T.F. Westbrook, K.K. Wong, and S.J. Elledge. 2009. A Genomewide RNAi screen identifies multiple synthetic lethal interactions with the Ras oncogene. Cell 137:835-848

10. Zuber, J., K. McJunkin, C. Fellmann, L.E. Dow, M.J. Taylor, G.J. Hannon, and S.W. Lowe. 2011. Toolkit for evaluating genes required for proliferation and survival using tetracycline-regulated RNAi. Nat. Biotechnol. 29:79-83

11. Scuoppo, C., C. Miething, L. Lindqvist, J. Reyes, C. Ruse, I. Appelmann, S. Yoon, A. Krasnitz, et al. 2012. A tumour suppressor network relying on the polyamine-hypusine axis. Nature 487:244-248.

12. Paddison, P.J., M. Cleary, J.M. Silva, K. Chang, N. Sheth, R. Sachidanandam, and G.J. Hannon. 2004. Cloning of short hairpin RNAs for gene knockdown in mammalian cells. Nat. Methods 1:163-167.

13. Dow, L.E., P.K. Premsrirut, J. Zuber, C. Fellmann, K. McJunkin, C. Miething, Y. Park, R.A. Dickins, et al. 2012. A pipeline for the generation of shRNA transgenic mice. Nat. Protoc. 7:374-393.

14. Tiscornia, G., O. Singer, and I.M. Verma. 2006. Production and purification of lentiviral vectors. Nat. Protoc. 1:241-245.

15. Blasco, R.B., S. Francoz, D. Santamaría, M. Cañamero, P. Dubus, J. Charron, M. Baccarini, and M. Barbacid. 2011. C-Raf, but not B-Raf, is essential for development of 
K-Ras oncogene-driven non-small cell lung carcinoma. Cancer Cell 19:652-663.

16. Guerra, C., N. Mijimolle, A. Dhawahir, P. Dubus, M. Barradas, M. Serrano, V. Campuzano, and M. Barbacid. 2003. Tumor induction by an endogenous K-ras oncogene is highly dependent on cellular context. Cancer Cell 4:111-120.

17. Belteki, G., J. Haigh, N. Kabacs, K. Haigh, K. Sison, F. Costantini, J. Whitsett, S.E. Quaggin, and A. Nagy. 2005. Conditional and inducible transgene expression in mice through the combinatorial use of Cre-mediated recombination and tetracycline induction. Nucleic Acids Res. 33:e51.

18. Loonstra, A., M. Vooijs, H.B. Beverloo, B.A. Allak, E. van Drunen, R. Kanaar, A. Berns, and J. Jonkers. 2001. Growth inhibition and DNA damage induced by Cre recombinase in mammalian cells. Proc. Natl. Acad. Sci. USA 98:9209-9214.

19. Premsrirut, P.K., L.E. Dow, S.Y. Kim, M. Camiolo, C.D. Malone, C. Miething, C. Scuoppo, J. Zuber, et al. 2011. A rapid and scalable system for studying gene function in mice using conditional RNA interference. Cell 145:145-158

20. Stern, P., S. Astrof, S.J. Erkeland, J. Schustak, P.A. Sharp, and R.O. Hynes. 2008. A system for Cre-regulated RNA interference in vivo. Proc. Natl. Acad. Sci. USA 105:13895-13900.

21. Cho, H., T. Herzka, W. Zheng, J. Qi, J.E. Wilkinson, J.E. Bradner, B.D. Robinson, M. Castillo-Martin, et al. 2014. RapidCaP, a novel GEM model for metastatic prostate cancer analysis and therapy, reveals Myc as a driver of Pten-mutant metastasis. Cancer Discov. 4:318-333.

22. Rodriguez, E., L. Mannion, P. D'Santos, M. Griffiths, M.J. Arends, K.M. Brindle, and S.K. Lyons. 2014. Versatile and enhanced tumour modelling in mice via somatic cell transduction. J. Pathol. 232:449-457.

23. Karreth, F.A., K.K. Frese, G.M. DeNicola, M. Baccarini, and D.A. Tuveson. 2011. C-Raf is required for the initiation of lung cancer by K-RasG12D. Cancer Discov. 1:128136.

24. Fellmann, C., J. Zuber, K. McJunkin, K. Chang, C.D. Malone, R.A. Dickins, Q. Xu, M.O. Hengartner, et al. 2011. Functional identification of optimized RNAi triggers using a massively parallel sensor assay. Mol. Cell 41:733-746.

25. DuPage, M., A.L. Dooley, and T. Jacks. 2009. Conditional mouse lung cancer models using adenoviral or lentiviral delivery of Cre recombinase. Nat. Protoc. 4:1064-1072.

26. Fernández-Medarde, A. and E. Santos. 2011. Ras in cancer and developmental diseases. Genes Cancer. 2:344-358.

27. Gysin, S., M. Salt, A. Young, and F. McCormick. 2011. Therapeutic strategies for targeting ras proteins. Genes Cancer 2:359-372.

28. Liu, Y., K. Marks, G.S. Cowley, J. Carretero, Q. Liu, T.J.F. Nieland, C. Xu, T.J. Cohoon, et al. 2013. Metabolic and functional genomic studies identify deoxythymidylate kinase as a target in LKB1-mutant lung cancer. Cancer Discov. 3:870-879.

29. Balbin, O.A., J.R. Prensner, A. Sahu, A. Yocum, S. Shankar, R. Malik, D. Fermin, S.M. Dhanasekaran, et al. 2013. Reconstructing targetable pathways in lung cancer by integrating diverse omics data. Nat Commun. 4:2617.

30. Holohan, C., S. Van Schaeybroeck, D.B. Longley, and P.G. Johnston. 2013. Cancer drug resistance: an evolving paradigm. Nat. Rev. Cancer 13:714-726.

31. Fellmann, C., T. Hoffmann, V. Sridhar, B. Hopfgartner, M. Muhar, M. Roth, D.Y. Lai, I.A. Barbosa, et al. 2013. An optimized microRNA backbone for effective singlecopy RNAi. Cell Rep. 5:1704-1713.

32. Chicas, A., X. Wang, C. Zhang, M. McCurrach, Z. Zhao, O. Mert, R.A. Dickins, M. Narita, et al. 2010. Dissecting the unique role of the retinoblastoma tumor suppressor during cellular senescence. Cancer Cell 17:376-387

33. Hsu, P.D., E. Lander, and F. Zhang. 2014. Development and applications of CRISPRCas9 for genome engineering. Cell 157:1262 1278.

34. Politi, K., P. Fan, R. Shen, M. Zakowski, and H.E. Varmus. 2010. Erlotinib resistance in mouse models of epidermal growth factor receptor-induced lung adenocarcinoma. Dis Model Mech. 3:111-119.

Received 29 April 2014; accepted 5 August 2014.

Address correspondence to David Santamaría, Spanish National Cancer Research Centre, Madrid, Spain. E-mail: dsantamaria@cnio.es

To purchase reprints of this article, contact: biotechniques@fosterprinting.com

\section{Unlimited Biolmaging}

Colorimetric

Bioluminescence

Fluorescence

Chemiluminescence Multiplex
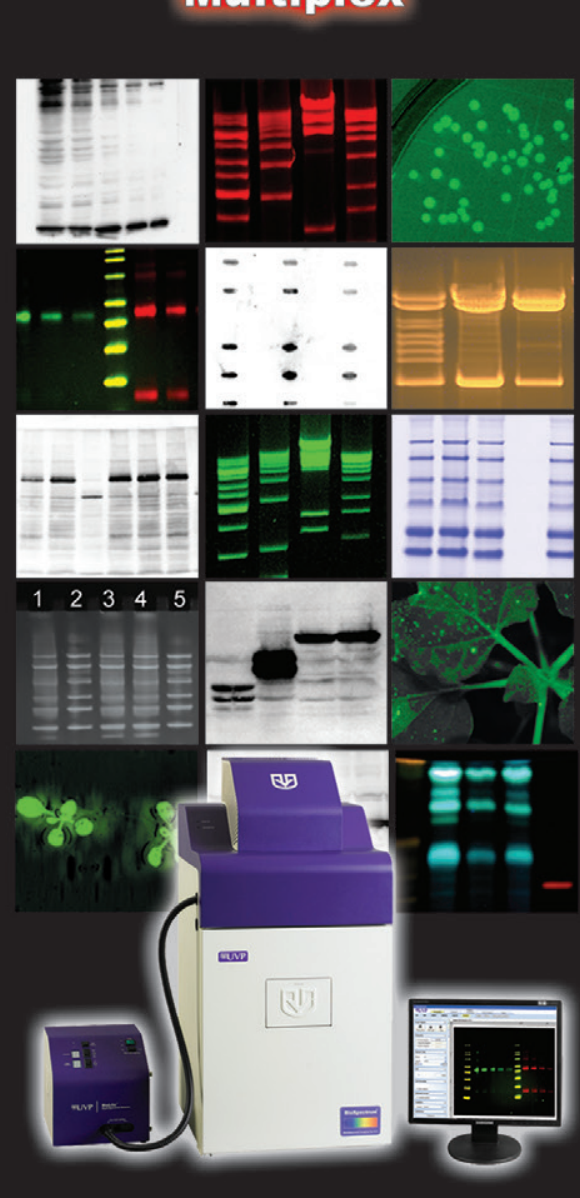

The BioSpectrum Imaging System with its advanced capabilities enables a wide range of imaging applications.

Unlimited in its ability to grow with your lab's developing life science research needs today and into the future.

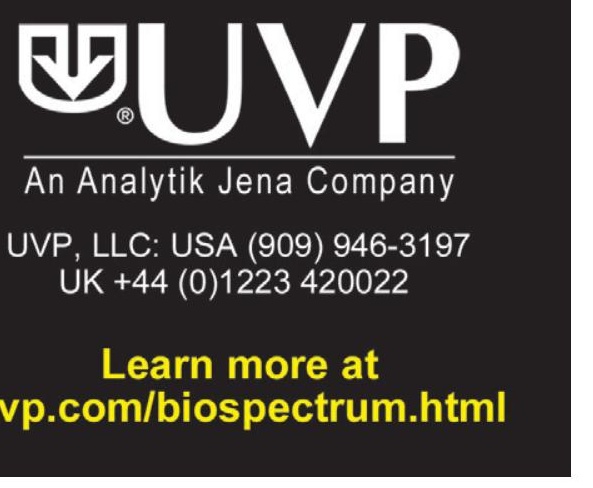

